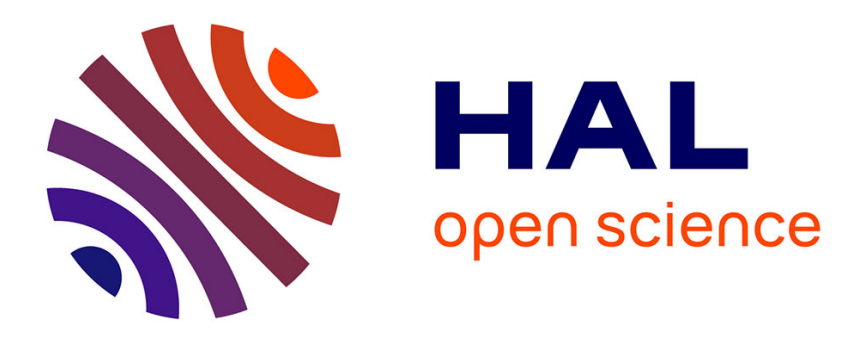

\title{
Phases of Talbot patterns in angular self-imaging
}

Hugues Guillet de Chatellus, Eric Lacot, Olivier Hugon, Olivier Jacquin, Naïma Khebbache, José Azaña

\section{To cite this version:}

Hugues Guillet de Chatellus, Eric Lacot, Olivier Hugon, Olivier Jacquin, Naïma Khebbache, et al.. Phases of Talbot patterns in angular self-imaging. Journal of the Optical Society of America. A Optics, Image Science, and Vision, 2015, pp.10.1364. 10.1364/AO.99.099999 . hal-01165168

\section{HAL Id: hal-01165168 https://hal.science/hal-01165168}

Submitted on 29 Jun 2015

HAL is a multi-disciplinary open access archive for the deposit and dissemination of scientific research documents, whether they are published or not. The documents may come from teaching and research institutions in France or abroad, or from public or private research centers.
L'archive ouverte pluridisciplinaire HAL, est destinée au dépôt et à la diffusion de documents scientifiques de niveau recherche, publiés ou non, émanant des établissements d'enseignement et de recherche français ou étrangers, des laboratoires publics ou privés. 


\title{
Phases of Talbot patterns in angular self- imaging
}

\author{
Hugues Guillet de Chatellus, ${ }^{1,2,{ }^{*}}$ Eric Lacot, ${ }^{1,2}$ Olivier Hugon, ${ }^{1,2}$ Olivier Jacquin, ${ }^{1,2}$ \\ Naïma Khebbache,$^{3}$ and José Azaña ${ }^{4}$ \\ ${ }^{1}$ Univ. Grenoble-Alpes, LIPhy, F-38000 Grenoble, France \\ ${ }^{2}$ CNRS, LIPhy, F-38000 Grenoble, France \\ ${ }^{3}$ Laboratory of Photonics Systems and Nonlinear Optics, Institute of Optics and Fine Mechanics, \\ University of Sétif, 19000 Algeria \\ ${ }^{4}$ INRS-Energie, Matériaux et Télécom., Montréal, Québec H5A1K6, Canada \\ *Corresponding author: hugues.guilletdechatellus@ujf-grenoble.fr
}

Received Month X, XXXX; revised Month X, XXXX; accepted Month X, XXXX; posted Month X, XXXX (Doc. ID XXXXX); published Month X, XXXX

\begin{abstract}
The original Talbot (self-imaging) effect is observed in the vicinity of a grating of slits shined with a plane wave, and results in periodic images of the initial diffraction pattern (integer Talbot effect) and the appearance of images with a periodicity reduced by an integer factor (fractional Talbot effect). Most of the studies on Talbot effect so far have focused on the distribution of the intensity of the diffracted light. However the phases of the Talbot images, obtained in both the integer and fractional self-imaging cases, can be calculated in a closed form and display interesting auto-correlation properties. This paper reports what we believe to be the first experimental investigation of the phases of Talbot images beyond the integer self-imaging case. We address the problem of experimental measurement of the phases of the Talbot images in the equivalent frame of the angular Talbot effect, a recently reported manifestation of the Talbot effect in the far field. The phases of the Talbot images are measured by far-field holography and the obtained results are in excellent agreement with theoretical calculations. They also suggest the possibility of using the scheme for a precise "fractional ruler" aimed at distances' measurements. (C) 2015 Optical Society of America

OCIS codes: (050.1950) Diffraction gratings; (070.6760) Talbot and self-imaging effects.

http://dx.doi/org/10.1364/AO.99.099999
\end{abstract}

\section{Introduction}

Since its discovery in optical diffraction [1, 2], Talbot effect has been observed and interpreted in various domains of wave physics, including quantum mechanics and electromagnetism in general [3-5]. Whereas the original Talbot effect was first observed and explained in the spatial domain, it was later observed in the temporal domain [6,7] and in the dual Fourier domains of spatial and temporal frequencies (angular and spectral Talbot effects, respectively) [8,9]. The most appealing features of the Talbot effect involve the possibility of creating an exact replica of a given periodic pattern (self-imaging, or integer Talbot effect) or the division of the periodicity of the original pattern by an integer factor (fractional Talbot effect). This property has led to applications in both the spatial domain (optical lithography and micropatterning [10]) and the temporal domain (generation of trains of light pulses with high repetition rate [6,7]). Essentially, the Talbot effect arises from the interference of waves with quadratic relative phases: in the original Talbot effect, the contributions of the individual slits of the grating to the intensity in the near field have a quadratic phase dependence in the paraxial approximation, while in the time domain, the Talbot effect arises typically in dispersive lines that imprint a quadratic spectral phase on the propagating pulses: the curvature of the parabola of the phases sets directly the specific integer or fractional Talbot pattern that is observed at the system output. Most of the practical applications of the Talbot effect so far make use of the intensity pattern, and the phase properties have triggered relatively limited interest, except for the case of Talbot array illuminators (TAIs).
TAIs take advantage of the possibility of generating a pure phase image from an amplitude grating and vice-versa, the possibility of creating an amplitude pattern from a pure phase grating [11-13]. In this context, the phases of the Talbot images have been studied theoretically, but the theoretical investigations have been restricted to a specific subset of fractional Talbot conditions [14]. Interestingly, the sequence of phases arising in fractional Talbot images correspond to the arguments of socalled Gauss sums, which in turn possess interesting autocorrelation properties $[15,16]$. For instance, considering that the phases of Talbot patterns constitute a set of orthogonal phase codes, they have been proposed as an alternative to Hadamard matrices for phase-encoded holographic multiplexing storage in volume-holographic media [17].

Despite their intrinsic fundamental interest and strong potential for information coding and processing applications, to the best of our knowledge, no experimental measurements of the phases of the Talbot images have been reported yet, except for certain integer Talbot selfimages and the observation of phase anomalies and Gouy phase shifts in the Talbot carpet $[18,19]$. Moreover since the original Talbot effect is essentially a near-field effect, the experimental investigation of the phases of the individual Talbot images can become quite challenging requiring for instance the use of a high resolution interference microscope [19].

In this paper, we extend the state of the art on the Talbot effect, by reporting a simple technique that enables a measurement of the phases 
of any fractional Talbot self-image. Practical limitations related to the intrinsic near-field nature of the conventional Talbot effect are overcome by carrying out the desired experimental characterization in an equivalent configuration, namely using the recently introduced angular Talbot effect. This effect can be interpreted as the counterpart of the spatial Talbot effect in the dual domain of angular frequencies i.e. observed in the far field [8]. The concept we use for the target phase measurements is derived from far-field holography, where the initial wavefront interferes with the Talbot image under analysis: the resulting intensity is then directly linked to the phase pattern of the Talbot image. As an additional consequence, our observations suggest a new concept for distance measurement using the employed interferometric scheme, referred here to as a "fractional ruler", where the distance between a point source and the diffraction grating is directly measured on the interference pattern, precisely expressed in the form of a fraction of two integers.

The manuscript is structured as follows: in the first section, we recall theoretical results on the classical near-field Talbot effect and focus especially on the phase of the Talbot images. Subsequently, we outline the direct equivalence between the original (near-field) and the angular (far-field) Talbot effects. In a third section, we present our measurement scheme of the phases of the angular Talbot images, and the experimental results, which are found to show an excellent agreement with our theoretical predictions.

\section{Theoretical aspects of the near-field Talbot effect}

In this part, we briefly recall the theory of the original (spatial) Talbot effect. Such effect can be observed in the problem of Fresnel diffraction of a monochromatic plane wave of wavelength $\lambda$ by a grating of a large number of slits $(\mathrm{N})$, ideally infinite along the direction $\mathrm{y}$, and periodically separated by a distance equal to $\Lambda$ along the direction of $\mathrm{x}$. We extend the theoretical treatment given in [20] by taking into account the envelope transmission function over the slits of the grating, $\mathrm{t}_{\mathrm{env}}(\mathrm{x})$. We define $\mathrm{g}(\mathrm{x})$ as the transmission of the grating over an elementary cell of the grating (i.e. $-\Lambda / 2<x<\Lambda / 2$ ). The grating is located in the plane $z=0$. Since the slits are infinite along $y$, the theoretical treatment can be restricted to the spatial variables $\mathrm{x}$ and $\mathrm{z}$ (diffraction distance).

\section{Grating transmission function}

The transmission function of the grating is given by:

$$
t(x)=t_{e n v}(x) \sum_{n} g(x-n \Lambda)
$$

and equivalently by:

$$
t(x)=\int g\left(x^{\prime}\right) \sum_{n} t_{e n v}(n \Lambda) \delta\left(x-x^{\prime}-n \Lambda\right) d x^{\prime}
$$

Eq. (2) is valid under the condition that the grating envelope function $\mathrm{t}_{\mathrm{env}}(x)$ is much slower than the individual grating aperture function $g(x)$, such that $\mathrm{t}_{\mathrm{env}}(x)$ is approximately constant along the individual grating aperture. Strictly, the angular frequency bandwidth of $t_{\text {env }}(x)$ must be much narrower than the angular frequency bandwidth of $g(x)$. Then:

The Fourier transform (FT) of $t(x)$ is: $T\left(k_{x}\right)=\int t(x) e^{i k_{x} x} d x$.

$$
T\left(k_{x}\right)=G\left(k_{x}\right) \sum_{n} t_{e n v}(n \Lambda) e^{i n k_{x} \Lambda}
$$

where $\mathrm{G}\left(\mathrm{k}_{\mathrm{x}}\right)$ and $\mathrm{T}_{\mathrm{env}}\left(\mathrm{k}_{\mathrm{x}}\right)$ are the Fourier transforms of $g(x)$ and $t_{\text {env }}(x)$ respectively. Defining $K_{0}=2 \pi / \Lambda$ the Poisson summation formula writes:

$$
\sum_{n} t_{e n v}(n \Lambda) e^{i n k_{x} \Lambda}=\sum_{m} T_{e n v}\left(k_{x}+m K_{0}\right)
$$

where the multiplicative constant has been dropped and finally:

$$
T\left(k_{x}\right)=G\left(k_{x}\right) \sum_{m} T_{e n v}\left(k_{x}+m K_{0}\right) .
$$

\section{Electric field in the Fresnel approximation}

Assuming that the incident plane wave propagates along $\mathrm{z}$, the electric field diffracted by the grating at location $(x, z)$ is proportional to the convolution product (represented by $*$ ) of the transmission function of the grating, by the impulse-response function of free space. In the Fresnel approximation, the latter can be written as:

$$
h_{z}(x)=h_{0} e^{-i k \frac{x^{2}}{2 z}} \text { where } h_{0}=i / \lambda z e^{-i k z} \text { and } k=\frac{2 \pi}{\lambda}[21] \text {. In }
$$
the spatial frequency domain, the transfer function of free space is:

$$
H_{z}\left(k_{x}\right)=H_{0} e^{i \lambda z k_{x}^{2} / 4 \pi} \text { where } H_{0}=e^{-i k z} \text {. Therefore, }
$$$$
E(x, z) \propto t(x) * h_{z}(x)=F T^{-1}\left(T\left(k_{x}\right) H_{z}\left(k_{x}\right)\right)
$$

which leads to:

$$
E(x, z) \propto H_{0} \int G\left(k_{x}\right) \sum_{m} T_{e n v}\left(k_{x}+m K_{0}\right) e^{\frac{i \lambda z k_{x}^{2}}{4 \pi}} e^{-i k_{x} x} d k_{x}
$$

Consistently with the above mentioned condition, Eq. (7) can be greatly simplified under the assumption that the function $\mathrm{G}\left(k_{x}\right)$ keeps approximately constant over the angular frequency bandwidth of $T_{e n v}\left(k_{x}\right)$. Moreover the quadratic phase term $\mathrm{e}^{i \lambda z k_{x}^{2} / 4 \pi}$ can also been approximated by $\mathrm{e}^{\mathrm{i} \pi \lambda \mathrm{z}^{2}} \frac{\mathrm{m}^{2}}{\Lambda^{2}}$ when $(\mathrm{m}-1 / \mathrm{N}) \mathrm{K}_{0}<k_{x}<(\mathrm{m}+1 / \mathrm{N}) \mathrm{K}_{0}$ ( $\mathrm{m}$ integer). Under these conditions, Eq. (7) can be expressed as follows:

$E(x, z) \propto t_{e n v}(x) e^{-i k z} \sum_{m} G\left(m K_{0}\right) e^{i \pi \lambda z \frac{m^{2}}{\Lambda^{2}}} e^{-i m K_{0} x}$

The usual Talbot carpet is obtained by plotting $|E(x, z)|^{2}$ in the $(x, z)$ plane from Eq. 8 (fig. 1, top). Another similarly interesting manifestation of the Talbot effect is the plot of the phase carpet in the $(x, z)$ plane (fig. 1, bottom).

\section{Observation of the Talbot effect}

We define the Talbot length as $z_{T}=\Lambda^{2} / \lambda$ and consider the intensity at a distance from the grating $z$ equal to a fraction $p / q$ of the Talbot length ( $p$ and $q$ being coprime integers). Then:

$E\left(x, \frac{p}{q} z_{T}\right) \propto t_{\text {env }}(x) e^{-i k_{\bar{q}}^{p} z_{T}} \sum_{m} G\left(m K_{0}\right) e^{i \pi \frac{p}{q} m^{2}} e^{-i m K_{0} x}$.

We now follow the standard procedure for analysis of the Talbot effect and define $\varphi(m)=\pi \frac{p}{q} m^{2}[20]$.

When the product $p q$ is even, $\varphi(m+q)=\varphi(m)$ while when $p q$ is odd, $\varphi(m+q)=\varphi(m)+\pi$. We regroup the terms having the same phase, modulo $\pi$ which leads to: 


$$
\begin{gathered}
E\left(x, \frac{p}{q} z_{T}\right) \propto t_{e n v}(x) e^{-i k \frac{p}{q} z_{T}} \times \\
\sum_{n=0}^{q-1} e^{i \pi \frac{p}{q} n^{2}} e^{-i n K_{0} x} \sum_{l} G\left((n+l q) K_{0}\right) e^{-i l p q \pi} e^{-i l q K_{0} x}
\end{gathered}
$$
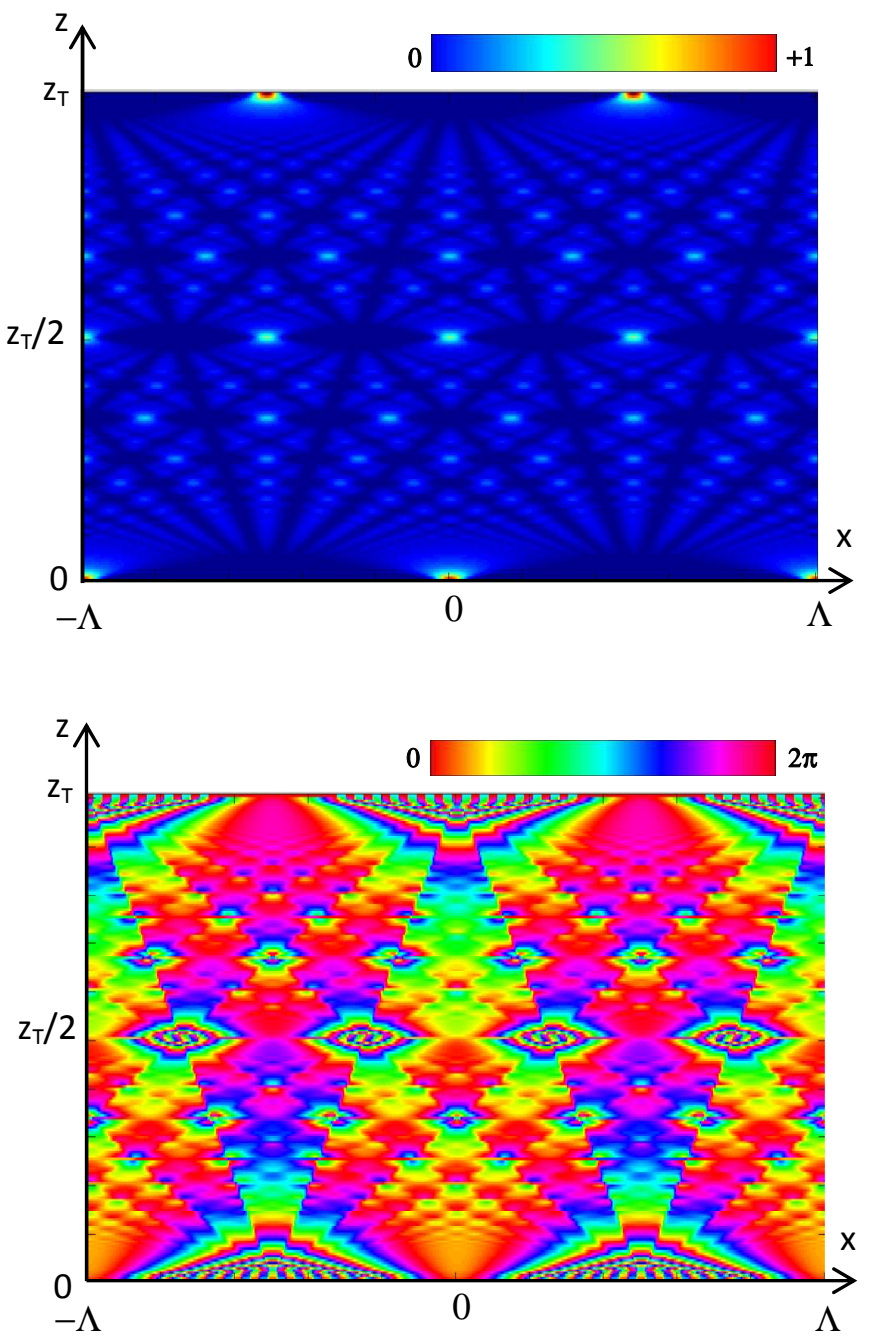

Fig. 1. Theoretical normalized intensity (top) and phase (bottom) Talbot carpets. A periodic grating of slits, centered at $\mathrm{x}=0$ and infinite along $\mathrm{x}$, is shined with a plane monochromatic wave. The transmission function of the slits $g(x)$ is a gaussian profile, the ratio between the width of the slits and the period of the grating is about $1 / 30$. Top: intensity plot as a function of $\mathrm{x}$ and $\mathrm{z}$ when $-\Lambda<\mathrm{x}<$ $\Lambda$ and $0<\mathrm{z}<\mathrm{z}_{\mathrm{T}}$ (with $z_{T}=\Lambda^{2} / \lambda$ ). The intensity values are normalized with respect to the maximum across the pattern. Bottom: longitudinal differential phase due to the Talbot effect: the quantity $\arg (\mathrm{E}(\mathrm{x}, \mathrm{z}))-\arg \left(\mathrm{E}_{0}(\mathrm{x}, \mathrm{z})\right)$ is plotted on the same boundary, $\mathrm{E}_{0}(\mathrm{x}, \mathrm{z})$ being the electric field of the incident plane-wave that would have propagated in the absence of the grating, i.e. $E_{0}(x, z) \propto e^{-i k z}$. The asymmetric "hourglass" shape at every Talbot spot (e.g. at $\mathrm{z}=\mathrm{Z \textrm {T }} / 2$ ) is the signature of the Gouy phase in a $1 \mathrm{D}$ geometry -or longitudinal $\pi / 2$-phase shift-, as recently reported in [18]. gives:

Replacing $G$ with its expression and dropping the first phase term
$E\left(x, \frac{p}{q} z_{T}\right) \propto t_{e n v}(x) \times$

$\sum_{n=0}^{q-1} e^{i \pi \frac{p}{q} n^{2}} e^{-i n K_{0} x} \int g\left(x^{\prime}\right) e^{i n K_{0} x^{\prime}} \sum_{l} e^{-i l q K_{0}\left(x-x^{\prime}\right)} e^{-i l p q \pi} d x^{\prime}$

The Poisson summation formula writes:

$\sum_{l} e^{-i l q K_{0}\left(x-x^{\prime}\right)} e^{-i l p q \pi}=\sum_{l} \delta\left(x^{\prime}-x+\frac{\pi}{K_{0}}\left(\frac{2 l}{q}-p\right)\right)$

Finally:

$$
\begin{gathered}
E\left(x, \frac{p}{q} z_{T}\right) \propto t_{e n v}(x) \times \\
\sum_{l} g\left(x-\Lambda\left(\frac{l}{q}-\frac{p}{2}\right)\right) \sum_{n=0}^{q-1} e^{i \frac{\pi}{q}\left[p n^{2}-2 n l+n p q\right]}
\end{gathered}
$$

The Gauss sum defined by the second summation can be evaluated in closed form depending on the parity of the integers $p$ and q. It can be shown that:

$$
\sum_{n=0}^{q-1} e^{i \frac{\pi}{q}\left[p n^{2}-2 n l+n p q\right]}=\sqrt{q} e^{i \Phi(l, q, p)}
$$

where the phasor $e^{i \Phi(l, q, p)}$ is given by the following expressions $[22,23]$.

When p even and q odd:

$\left(\begin{array}{l}p \\ q\end{array}\right) \exp \left(-i \frac{\pi}{4}\left[(q-1)+4 \frac{p}{q}\left(\left[\frac{1}{p}\right]_{q}\right)^{2} l^{2}\right]\right)$

when p odd and q odd:

$$
\left(\begin{array}{l}
p \\
q
\end{array}\right) \exp \left(-i \frac{\pi}{4}\left[(q-1)+8 \frac{p}{q}\left[\frac{1}{2}\right]_{q}\left(\left[\frac{1}{2 p}\right]_{q}\right)^{2}(2 l+q)^{2}\right]\right)
$$

when p odd and q even:

$\left(\begin{array}{l}q \\ p\end{array}\right) \exp \left(-i \frac{\pi}{4}\left[-p+\frac{p}{q}\left(\left[\frac{1}{p}\right]_{q}\right)^{2}(2 l+q)^{2}\right]\right)$

where $\left[\frac{1}{a}\right]_{b}$ is the inverse of a modulo $\mathrm{b}$ and $\left(\begin{array}{l}a \\ b\end{array}\right)$ is the Jacobi symbol equal to +1 if there is an integer $\mathrm{m}$ so that $m^{2}=a[b]$ and -1 otherwise. Interestingly the phases of the Talbot images show a quadratic dependence with the output individual aperture position, identified by the factor $l$. This is intimately linked to the fact that the Talbot images result themselves from the interference of waves with quadratic relative phases and that the Fourier transform of a linear chirp (quadratic phase) is also a linear chirp.

\section{Correlation properties of the phases of Talbot images}

Another interesting property of these phases appears when one considers the complex sequence defined by $r_{1}=e^{i \Phi(l, q, p)}$. The sequence is q-periodic and it can be shown that the autocorrelation of the sequence, given by $c_{n}=\sum_{l=0}^{q-1} r_{l} r_{l+n}^{*}$ is equal to $q \delta(n \bmod q)$, where $\delta(n \bmod q)$ is equal to unity when $\mathrm{n}$ is a multiple of $\mathrm{q}$, and 0 otherwise [16]

A simple physical proof can be given for this relation: since the Talbot image is the image of the grating after free space propagation in 
the near field, the intensity diffracted in the far field from the Talbot image is identical to the intensity diffracted in the far field by the initial grating. Considering a grating made of a large number of slits of negligible width and shined with a plane wave at normal incidence, the intensity diffracted in the far-field is simply:

$$
I\left(k_{x}\right) \propto \sum_{l} \delta\left(k_{x}-l K_{0}\right)=\sum_{n} e^{i 2 \pi n k_{x} / K_{0}} .
$$
writes:

The Wiener-Khinchin theorem for the intensity at $z=(p / q) z_{T}$

$$
I\left(k_{x}\right) \propto F T\left[E\left(x, \frac{p}{q} z_{T}\right) * E^{*}\left(x, \frac{p}{q} z_{T}\right)\right]
$$

where we recall that * stands for convolution. Straightforward calculations lead to:

$$
I\left(k_{x}\right) \propto \sum_{n} e^{i 2 \pi n k_{x} / q K_{0}} \sum_{l} r_{l} r_{l+n}^{*} .
$$

The equivalence between Eq. 18 and Eq. 20 implies that the autocorrelation $\sum_{1} r_{l} r_{l+n}^{*}$ vanishes except when $n$ is a multiple of $q$. Therefore the sequence of the phases occurring in the Talbot images is an example of delta-correlated discrete sequences, which have found applications in holography [17], acoustics, coding and signal compression [15].

\section{Talbot effect}

Despite their appealing properties, to our knowledge, no experimental measurement of the phases of Talbot images has been provided yet, beyond the integer self-imaging case. One reason is that the classical Talbot effect is a near-field effect: indeed any interferometric method requires mixing the Talbot image with a reference light beam, e.g. the incident light field, which implies a certain level of complexity [19].

Here we propose to measure the phases of the Talbot image by using a recently introduced, novel configuration, called "angular Talbot effect", which involves an equivalent manifestation of the Talbot effect in the reciprocal spatial frequency domain [8]. The advantage over the classical Talbot effect is that it manifests in the far field, which inherently makes the investigation of the phases by a far field holography technique more practical and flexible.

In the angular Talbot effect, the grating of slits is shined with a point-like light source located at a specific distance $\mathrm{d}$ from the grating. In the paraxial approximation, the phase profile of the wave that has propagated from the source to the plane of the grating has a parabolic (quadratic) shape. The intensity diffracted in the far field by the grating (Fraunhofer approximation) results from the interference of waves with quadratic relative phase shifts, leading to a Talbot effect in the far field. More precisely, the angular Talbot effect can also been understood as a self-imaging effect in the dual space of transverse wave-vector (or angular frequencies) [8]. The field diffracted in the far-field by the grating shined with a point-like source is:

$$
\tilde{E}\left(k_{x}, k_{y}\right) \propto F T\left(t(x) h_{d}(x)\right) e^{i \frac{\lambda d}{4 \pi} k_{y}^{2}} .
$$

Interestingly, the $\mathrm{x}$-dependent term $\widetilde{\mathrm{E}}\left(\mathrm{k}_{\mathrm{x}}\right) \propto \mathrm{FT}\left(\mathrm{t}(\mathrm{x}) \cdot \mathrm{h}_{\mathrm{d}}(\mathrm{x})\right)$ is simply the angular-frequency counterpart of Eq. 6 . The derivation of the diffracted field is relatively straightforward: since the space variables $\mathrm{x}$ and $\mathrm{y}$ are separated, $\widetilde{\mathrm{E}}\left(\mathrm{k}_{\mathrm{x}}\right)$ can be evaluated in the plane $\mathrm{y}=0$.
The field at the grating location $(\mathrm{x}, 0,0)$ is proportional to:

$$
E(x, 0,0) \propto t(x) h_{d}(x)
$$

which leads to:

$E(x, 0,0) \propto t_{e n v}(x) \int g\left(x^{\prime}\right) \sum_{n} \delta\left(x-x^{\prime}-n \Lambda\right) e^{-i \frac{\pi}{\lambda} \frac{n^{2} \Lambda^{2}}{d}} d x$

and:

$$
\begin{aligned}
& E(x, 0,0) \propto \\
& \int g\left(x^{\prime}\right) \sum_{n} \delta\left(x-x^{\prime}-n \Lambda\right) t_{e n v}(n \Lambda) e^{-i \frac{\pi}{\lambda} \frac{n^{2} \Lambda^{2}}{d}} d x^{\prime}
\end{aligned}
$$

where we assume that the phase of the wave incident on the grating can be considered as a constant over the extension of a single slit. Note that the reference of the phases is taken at the slit \#0 (i.e. at $\mathrm{x}$ $=0$ ). The Fourier-transform of the electric field in the plane of the grating is:

$$
\tilde{E}\left(k_{x}\right) \propto G\left(k_{x}\right) \int \sum_{n} \delta(x-n \Lambda) t_{e n v}(n \Lambda) e^{-i \frac{\pi}{\lambda} \frac{n^{2} \Lambda^{2}}{d}} e^{i k_{x} x} d x
$$

And finally:

$$
\tilde{E}\left(k_{x}\right) \propto G\left(k_{x}\right) \sum_{n} t_{e n v}(n \Lambda) e^{i n k_{x} \Lambda} e^{-i \frac{\pi}{\lambda} \frac{n^{2} \Lambda^{2}}{d}} .
$$

This result is mathematically equivalent to the form of Eq. 8, by properly exchanging the $\mathrm{x}$ and $\mathrm{k}_{\mathrm{x}}$ variables, which allows one to draw $\mathrm{a}$ formal parallelism between the classical and the angular Talbot effect cases. By analogy with the above discussions on the observation of the Talbot effect in the classical near-field case, the condition for angular Talbot effect corresponds to a condition on the distance between the point-like source and the plane of the grating. In particular, when $d=\frac{m}{s} Z_{T}\left(z_{T}=\Lambda^{2} / \lambda\right)-m$ and $s$ - being coprime integers, the far field diffraction pattern shows a multiplication of the diffraction orders (or division of the angular distance between consecutive diffraction orders) by a factor of $m$ as compared to the case where the wave incident on the grating is a plane wavefront. More precisely, by directly transferring here the results obtained for the classical Talbot effect, when $d=\frac{m}{s} Z_{T}$, the diffracted field is proportional to:

$$
\begin{gathered}
\tilde{E}\left(k_{x}\right) \propto G\left(k_{x}\right) \times \\
\sum_{l} T_{e n v}\left(k_{x}-K_{0}\left(\frac{l}{m}-\frac{s}{2}\right)\right) \sum_{n=0}^{m-1} e^{-i \frac{\pi}{m}\left[s n^{2}-2 n l+n m s\right]}
\end{gathered}
$$

which is again formally equivalent to Eq. 13 . In particular, the phase of the wave diffracted along the transverse wave vector $\mathrm{k}_{\mathrm{x}}=K_{0}\left(\frac{l}{m}-\frac{s}{2}\right)$ is simply $-\Phi(l, m, s)$. Using similar procedures to those outlined above for the classical Talbot effect, both intensity and phase carpets can be calculated in the angular case as well (Fig. 2). Notice that despite the formal equivalence between near-field and angular Talbot effects, the carpets plotted on Fig. 1 and Fig. 2 differ, due to the fact that the angular Talbot carpets are plotted as a function of $d$, and not of $1 / d$, which is the formal equivalent, in the angular case, of $z$ in the near-field case. 

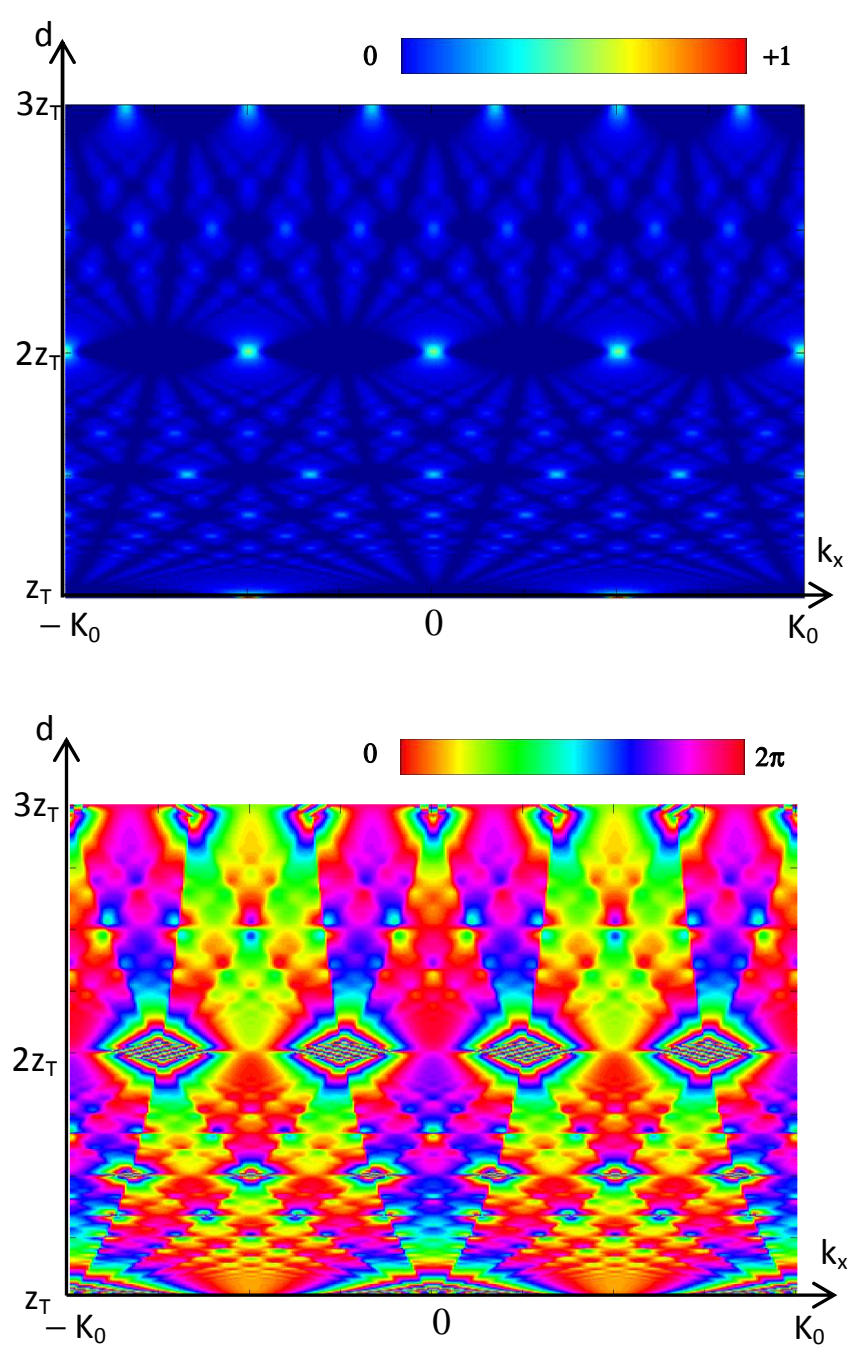

Fig. 2. Theoretical normalized intensity (top) and phase (bottom) angular Talbot carpets. A periodic grating of 51 slits with negligible width and a Gaussian envelope transmission function centered at $\mathrm{x}=0$, is shined with a point-like source located at a distance $\mathrm{d}$ from the grating. Top: intensity plot as a function of $\mathrm{k}_{\mathrm{x}}$ and $\mathrm{d}$ when $-\mathrm{K}_{0}<\mathrm{k}_{\mathrm{x}}<\mathrm{K}_{0}$ and $\mathrm{z}_{\mathrm{T}}<\mathrm{d}<3 \mathrm{z}_{\mathrm{T}}$ (with $z_{T}=\Lambda^{2} / \lambda$ ). Recall that if the grating were illuminated with a plane wave propagating along $\mathrm{z}$, the light would be diffracted along the directions $\mathrm{k}_{\mathrm{x}}=\mathrm{nK} \mathrm{N}_{0} \mathrm{n}$ integer. Bottom: phase distribution in the angular Talbot effect (plot of $\arg \left(\tilde{E}\left(k_{x}\right)\right.$ ).

\section{Measurement of the phases in the Talbot angular effect}

\section{Principle}

The possibility to observe the Talbot effect in the far field enables to measure the phases of the Talbot pattern in a simpler manner since in this case, it is relatively easier to 'compare' the diffracted waves under analysis with a spherical reference wave, using a similar strategy to that of far-field holography [24]. We consider a grating of slits shined with a point-like source $S$ located at a distance $d$ from the grating.

A virtual reference point-like source $S^{\prime}$ coherent with the primary source $\mathrm{S}$, is vertically shifted from $\mathrm{S}$, so that $\mathrm{SS}^{\prime}=\mathrm{a}$. The field emitted by
$\mathrm{S}^{\prime}$ in the direction $\left(\mathrm{k}_{\mathrm{x}}, \mathrm{k}_{\mathrm{y}}\right)$ at the object plane (distance $\mathrm{d}$ from the source) is given by:

$$
\tilde{E}_{r e f}\left(k_{x}, k_{y}\right) \propto e^{i\left[a k_{y}+\frac{\lambda d}{4 \pi}\left(k_{x}^{2}+k_{y}^{2}\right)\right]}
$$

where the reference of the phases is chosen at $\Omega(\mathrm{x}=\mathrm{y}=0)$ (Fig. 3).

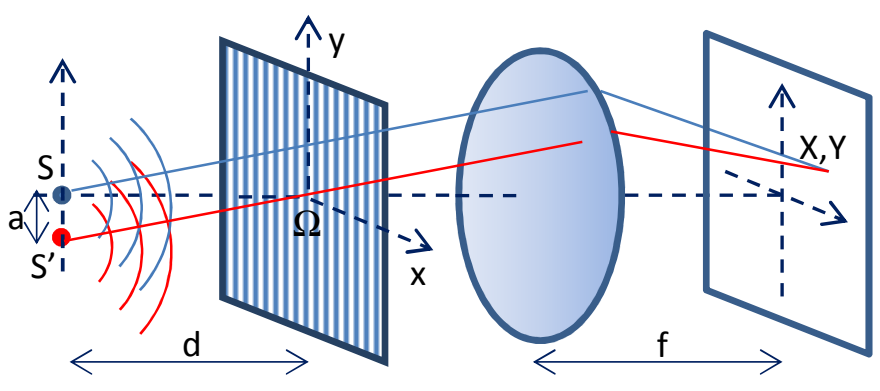

Fig. 3. Principle of the holographic measurement of the phases of the Talbot images in the configuration of the angular Talbot effect. The light diffracted by the grating of slits shined with the point-like source (blue), interferes with the reference (undiffracted) spherical wave produced by the virtual point-like source $S^{\prime}$ (red) in the far field (i.e. in the focal plane of a converging lens).

On the other hand, when the angular Talbot condition is satisfied, i.e., $d=\frac{m}{s} Z_{T}\left(z_{T}=\Lambda^{2} / \lambda\right)$, then the field diffracted by the grating in the direction $\left(\mathrm{k}_{\mathrm{x}}, \mathrm{k}_{\mathrm{y}}\right)$ is, according to Eq. 21 and Eq. 27:

$$
\begin{aligned}
& \tilde{E}\left(k_{x}, k_{y}\right) \propto G\left(k_{x}\right) \times \\
& \quad\left(\sum_{l} T_{e n v}\left(k_{x}-K_{0}\left(\frac{l}{m}-\frac{s}{2}\right)\right) e^{-i \Phi(l, m, s)}\right) e^{i \frac{\lambda d}{4 \pi} k_{y}^{2}}
\end{aligned}
$$

When the amplitude of the reference wave matches the amplitude of the wave diffracted in the direction $\left(\mathrm{k}_{\mathrm{x}}=K_{0}\left(\frac{l}{m}-\frac{s}{2}\right), \mathrm{k}_{\mathrm{y}}\right)$, the resulting intensity of $\tilde{E}\left(k_{x}, k_{y}\right)+\tilde{E}_{r e f}\left(k_{x}, k_{y}\right)$ is proportional to:

$$
1+\cos \left(\Phi(l, m, s)+a k_{y}+\frac{1}{4 \pi} \lambda d k_{x}^{2}+\phi_{0}\right)
$$

where $\phi_{0}$ is a constant phase shift. When the diffraction pattern is observed in the focal plane of a converging lens of focal length $\mathrm{f}$, one obtains an image of the angular energy spectrum in Eq. (30), so that the coordinates $(X, Y)$ of a point at the focal plane are linked to the transverse coordinates of the wave-vector $\left(\mathrm{k}_{\mathrm{x}}, \mathrm{k}_{\mathrm{y}}\right)$ by $\mathrm{X}=\frac{\lambda \mathrm{f}}{2 \pi} \mathrm{k}_{\mathrm{x}}$ and $Y=\frac{\lambda f}{2 \pi} k_{y}$. The aim of vertically shifting the virtual reference source is to record a continuous variation of the phase on the screen. It is worth noting that even though the phases of the Talbot images are m-periodic as a function of $l$, the recorded relative phase patterns are s-periodic. This is due to the fact the described interferometric set-up does not measure directly $\Phi(\mathrm{l}, \mathrm{m}, \mathrm{s})$ : additional terms involving $\mathrm{l}, \mathrm{s}$ and $\mathrm{m}$ appear in Eq. 30 and it is simple to check that the resulting theoretical expression are in fact s-periodic (Fig. 4). For instance, in the case where 
$\mathrm{s}$ is even and $\mathrm{m}$ is odd, the difference beween the relative phases of Talbot images $l+\mathrm{s}$ and $l$ writes:

$$
\begin{gathered}
\Delta \Phi=\Phi(l+s, m, s)-\Phi(l, m, s)+\pi \frac{m}{s}\left(\frac{l+s}{m}-\frac{s}{2}\right)^{2} \\
-\pi \frac{m}{s}\left(\frac{l}{m}-\frac{s}{2}\right)^{2} .
\end{gathered}
$$

Then:

$$
\Delta \Phi=-2 \pi \frac{l s^{2}}{m}\left(\left[\frac{1}{s}\right]_{m}\right)^{2}-\pi \frac{s^{3}}{m}\left(\left[\frac{1}{s}\right]_{m}\right)^{2}+2 \pi \frac{l}{m}-\pi s+\pi \frac{s}{m}
$$

which vanishes (modulo $2 \pi$ ). The calculation is similar for the two other cases.

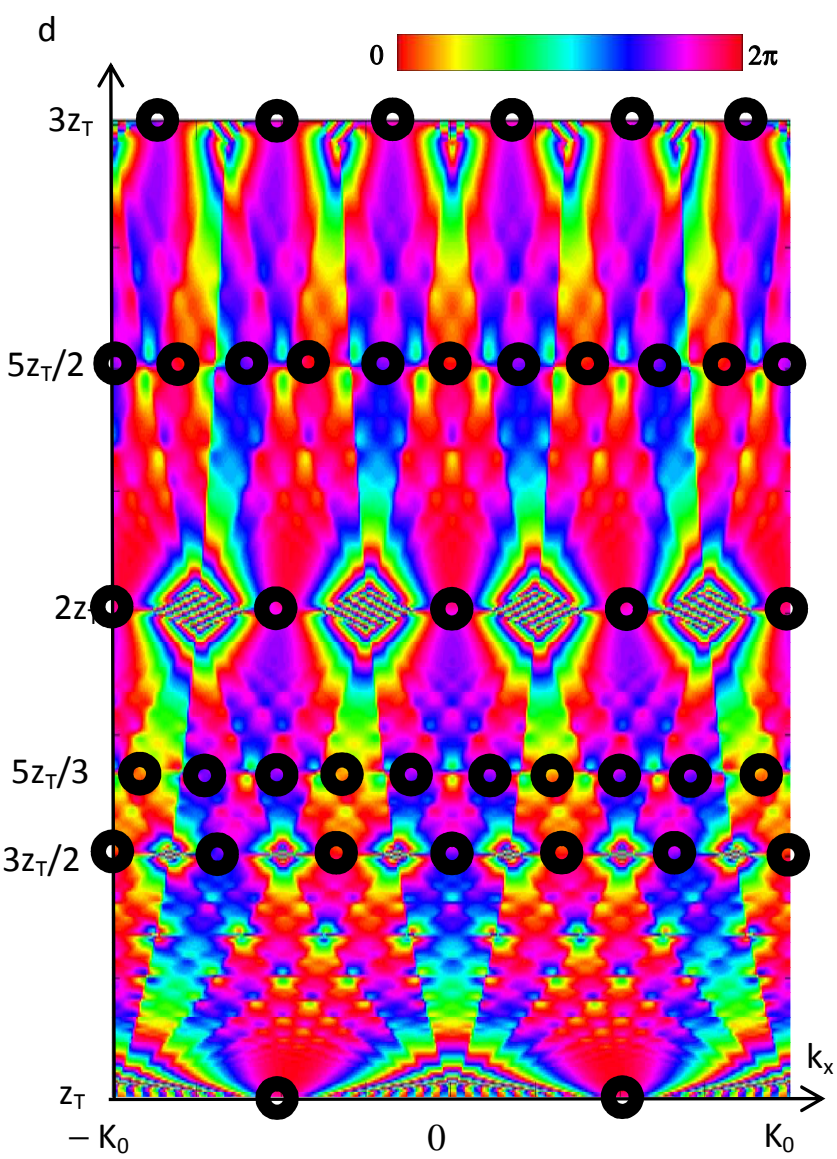

Fig. 4. Differential angular Talbot phase carpet: the quantity $\arg \left(\tilde{E}\left(k_{x}, 0\right)\right)$ $\arg \left(\tilde{E}_{r e f}\left(k_{x}, 0\right)\right)$ is plotted on the same boundary as Fig. 2 . The grating is the same as in Fig. 2. The light diffracted in the far-field interferes with the virtual spherical wave emitted by $S$ (in this case $S^{\prime}=S$ ). The black circles indicate the directions of the Talbot images in the cases $d=\mathrm{z}_{\mathrm{T}}, 3 \mathrm{Z}_{\mathrm{T}} / 2,5 \mathrm{Z}_{\mathrm{T}} / 3,2 \mathrm{Z}_{\mathrm{T}} 5 \mathrm{Z}_{\mathrm{T}} / 2$, and 3 Zт. Note that for any $\mathrm{d}=\mathrm{mZT} / \mathrm{s}$, the phases are s-periodic while the angular separation of the diffraction orders is divided by $\mathrm{m}$ ( $\mathrm{m}$ and $\mathrm{s}$ being coprime integers).

\section{Experimental setup}

The possibility to observe the Talbot effect in the far field renders the measurement of the phases of the Talbot images by interference techniques more practical and flexible. The experimental set-up is a classical Mach-Zehnder interferometer tuned near optical contact. A He-Ne laser (wavelength $\lambda=632.8 \mathrm{~nm}$ ) is sent into a microscope objective mounted on a translation stage. The resulting (spherical) wave is split and then recombined as in the usual Mach-Zehnder interferometer. A mirror in one of the arms is slightly tilted around the horizontal axis. Then the resulting interference pattern at the output of the interferometer observed at the focal plane of a converging lens (focal length $f$ ), consists in a succession of horizontal fringes with a fringe spacing equal to $\lambda f / a$, where $a$ is the distance between the two secondary point-like sources induced by the tilt of the mirror, similarly to conventional Young's double hole experiment (fig. 5, top). Then the grating of slits is inserted into the measurement arm of the interferometer (fig. 5, bottom).

a)

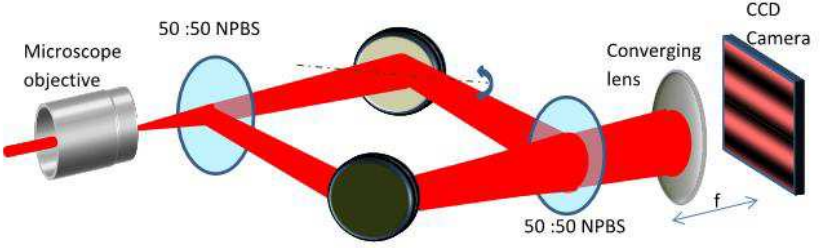

b)

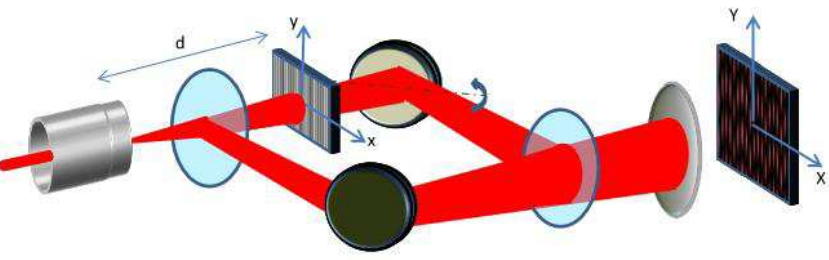

Fig. b. Sketch of the experimental set-up tor the measurement of the phases of the Talbot images (see text). NPBS stands for non-polarizing beam-splitter.

The grating is made with 50 slits (width: $7 \mu \mathrm{m}$ ) and the spatial period is $\Lambda=70 \mu \mathrm{m}$. The distance $\mathrm{d}$ between the point-like source and the grating can be adjusted by translating the grating. The intensity of the light pattern (hologram) is recorded on a CCD camera placed in the focal plane of the converging lens of focal length $\mathrm{f}=20 \mathrm{~cm}$. A rotating density filter (not shown on fig. 5) enables to control the intensity of the reference wave in order to maximize the contrast of the pattern recorded by the CCD camera. The distance $d$ between the point source and the grating is set to match a fraction of the Talbot length (i.e. $d=\frac{m}{s} Z_{T}$ where $z_{T}=7.8 \mathrm{~mm}$ ). The results are shown on fig. 6 and show a good agreement with the theoretical predictions, illustrating the practicability and accuracy of the technique.

As predicted by the theoretical description of the angular Talbot effect, the angular separation of the diffracted beams (along the horizontal $\mathrm{x}$ direction) is inversely proportional to $\mathrm{m}$. Interestingly, the intrinsic quadratic dependence of the phase profile of the Talbot image with l (Eq. 15 to 17) manifests by a parabolic shape of the interference pattern recorded on the CCD camera (especially visible in the cases $\mathrm{m} / \mathrm{s}$ $=6 / 5$ or $9 / 5$ in fig. 6 ).

Finally, as predicted theoretically, the phase pattern measured with this method is s-periodic. A surprising consequence of this property is that the values of $m$ and $s$ can be directly deduced from the 
two images recorded by the CCD, namely from the multiplicative factor in the intensity diffraction pattern and from the periodicity of the interference pattern, respectively. Based on this observation, this setup can be interpreted as a "fractional ruler": a precise fractional expression of the distance between the point source and the grating in units of $z_{T}$ can be deduced directly from the recorded CCD images. This technique differs significantly from standard optical techniques for distance measurement, where the detected signal varies with the distance to be measured in a continuous manner. In contrast, in the newly proposed method, diffraction pattern changes are observed in an entirely discrete fashion. Moreover, the result of the measurement is not a numerical value directly related to the distance of interest but consists of two coprime integers, whose ratio provides a precise estimate of the fractional distance to be measured - in units of the Talbot length.

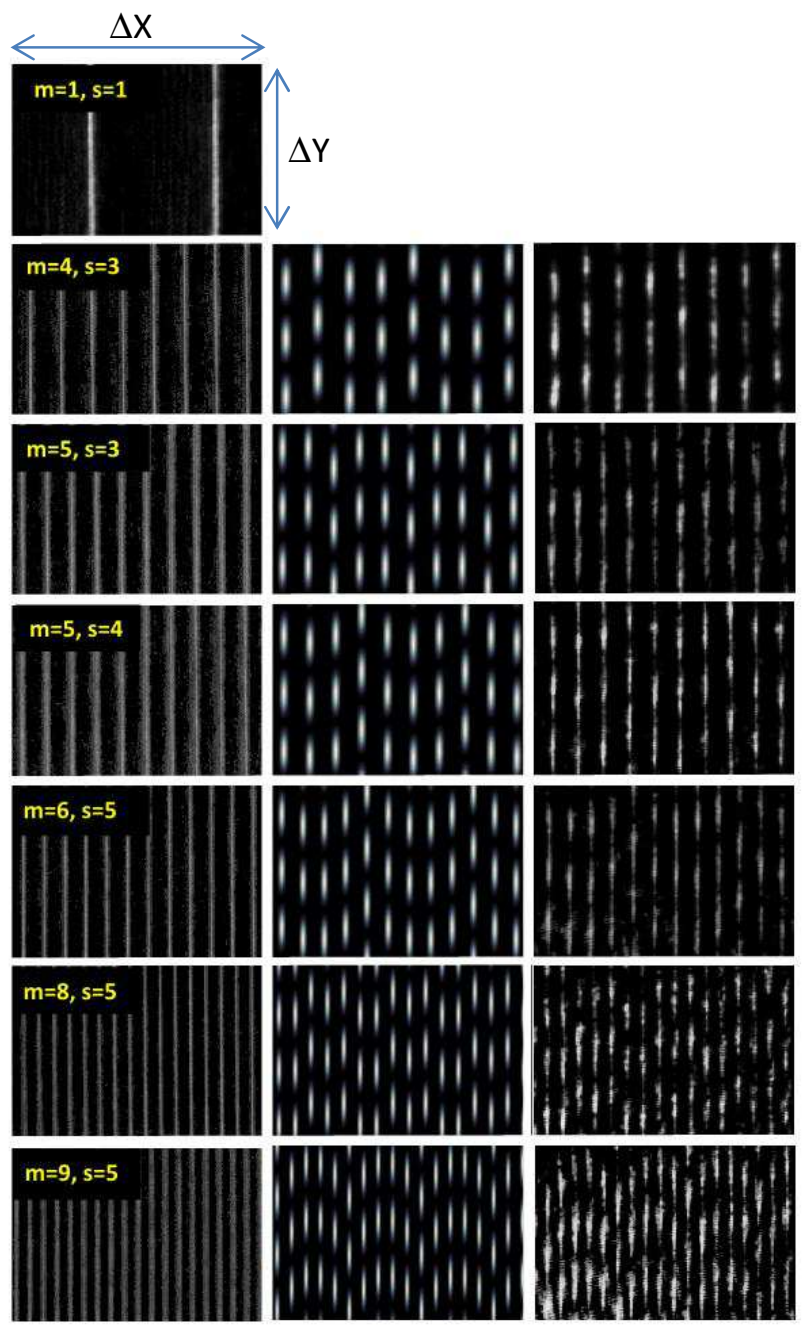

Fig. 6. Comparison between the theoretical and the experimental phase measurement for different values of $d$. Left column: experimental diffraction patterns in the angular Talbot effect (the reference arm is blocked). Middle (resp. right) column: theoretical (resp. experimental) phase images - or holograms. The size of the image recorded by the CCD camera in the focal plane of the converging lens corresponds to $\Delta \mathrm{Y}=3 \lambda \mathrm{f} / \mathrm{a}$ in the vertical direction (i.e. $\Delta \mathrm{k}_{\mathrm{y}}=6 \pi / \mathrm{a}$ ) and to $\Delta \mathrm{X}=\lambda \mathrm{fK}_{0} / \pi$ (i.e. $\Delta \mathrm{k}_{\mathrm{x}}=2 \mathrm{~K}_{0}$ ) in the horizontal direction. The contrast of both theoretical and experimental phase images has been numerically optimized for better visualization.
Conclusion

In this paper we have provided a detailed analysis of the parallelism between the original Talbot effect, which occurs in the nearfield diffraction region of a grating shined with a plane wave, and the recently demonstrated angular Talbot effect, which is observed in the far-field of a grating illuminated by a spherical wavefront. This analogy has allowed us to perform what we believe to be a first measurement of the phases of both integer and fractional Talbot images. Indeed, the angular Talbot effect offers a practical mean to carry out this measurement by far field holography. Moreover, our results suggest the possibility of using the grating-based interferometry set-up described here as a "fractional ruler", capable of providing a direct, precise fractional expression of the distance between the point source and the grating. This novel, interesting concept represents another illustration of the fascinating features of the Talbot effect.

\section{Acknowledgements}

HGC acknowledges the Commission Permanente de Coopération Franco-Québecoise (Call for grants PEES 2013), the Région RhôneAlpes (Program C-MIRA Explora Pro 2014) and the EMT-INRS for their financial support. NK acknowledges the Institute of Optics and fine Mechanics (Sétif, Algeria) for its financial support.

\section{References}

1. W. H. F. Talbot, "Facts relating to optical sciences," No. IV, Philos. Mag. 9, 401- 407 (1836).

2. Lord Rayleigh, "On copying diffraction gratings and on some phenomenon connected therewith," Philos. Mag. 11, 196-205 (1881).

3. J. Wen, Y. Zhang, and M. Xiao, "The Talbot effect: recent advances in classical optics, nonlinear optics, and quantum optics," Adv. Opt. Photon. 5, 83-130 (2013).

4. M. R. Dennis, N. I. Zheludev, and F. Javier Garcia de Abajo, “The plasmon Talbot effect," Opt. Exp. 15, 9692-9700 (2007)

5. L. Deng, E. W. Hagley, J. Denschlag, J. E. Simsarian, M. Edwards, C. W. Clark, K. Helmerson, S. L. Rolston, and W. D. Phillips, "Temporal, matter-wave-dispersion Talbot effect," Phys. Rev. Lett. 83, 5407-5411 (1999).

6. J. Azaña and M. A. Muriel, "Temporal self-imaging effects: theory and application for multiplying pulse repetition rates," IEEE J. Select. Topics Quantum Electron. 7, 728-744 (2001).

7. H. Guillet de Chatellus, O. Jacquin, O. Hugon, W. Glastre, E. Lacot, J. Marklof, "Generation of utra-high and tunable repetition rates in CW injection-seeded frequency shifted feedback lasers", Opt Exp. 21, 15065-15074 (2013).

8. J. Azaña, H. Guillet de Chatellus, "Angular Talbot Effect," Phys. Rev. Lett. 112, 213902 (2014).

9. J. Azaña, C. Wang, R. Chen, "Spectral self-imaging phenomena in sampled Bragg gratings", J. Opt. Soc. Am. B 22, 1829-1841 (2005). 
10. A. Isoyan, F. Jiang, Y. C. Cheng, F. Cerrina, P. Wachulak, L. Urbanski, J. Rocca, C. Menoni, and M. Marconi, "Talbot lithography: self-imaging of complex structures", J. Vac. Sci. Technol. B 27, 2931-2937 (2009).

11. A. W. Lohmann and J. A. Thomas, "Making an array illuminator based on the Talbot effect," Appl. Opt. 29, 43374340 (1990).

12. W. Klaus, Y. Arimoto, and K. Kodate "Talbot array illuminators providing spatial intensity and phase modulation" J. Opt. Soc. Am. A 14, 5 (1997).

13. P. Szwaykowski and V. Arrizon, "Talbot array illuminator with multilevel phase gratings," Appl. Opt. 32, 1109-1114 (1993).

14. C. Zhou, L. Liu, "Simple equations for the calculation of a multilevel phase grating for Talbot array illumination" Optics Comm. 115, 40-44 (1995).

15. M. R. Schroeder, "Number Theory in Science and Communications," 5th ed. (Springer, Berlin, Heidelberg, 2009), Chap. 28.

16. H. Guillet de Chatellus, O. Jacquin, O. Hugon, E. Lacot, "Theory of Talbot lasers," Phys. Rev. A 88, 033828 (2013).

17. C. Zhou, S. Stankovic, C. Denz , and T. Tschudi, "Phase codes of Talbot array illumination for encoding holographic multiplexing storage" Optics Comm. 161, 209-211 (1999).

18. M.-S. Kim, T. Scharf,, C. Menzel, C. Rockstuhl, and H. P. Herzig, "Phase anomalies in Talbot light carpets of self-images", Opt. Lett. 21, 1, 1287-1300 (2013).

19. M.-S. Kim, T. Scharf, C. Menzel, C. Rockstuhl, and H.P. Herzig, "Talbot images of wavelength-scale amplitude gratings", Opt. Expr., 20, 4903-4920 (2012).

20. M. V. Berry, and S. Klein, "Integer, fractional and fractal Talbot effects", J. Mod. Opt. 43, 2139-2164 (1996).

21. B. E. A. Saleh and M. C. Teich, "Fundamentals of Photonics," (John Wiley \& Sons, Inc., New York, USA, 1991), Chap. 4.

22. H. C. Rosu, J. P. Treviño, and H. Cabrera, "Talbot effect for dispersion in linear optical fibers and a wavelet approach," Int. J. Mod. Phys. B 20, 1860-1876 (2006).

23. S. Matsutani, and Y. Onishi, "Wave-particle complementarity and reciprocity of Gauss sums on Talbot effects," Found. Of Phys. Lett. 16, 325-341 (2003).

24. P. Hariharan, "Optical Holography: Principles, Techniques and Applications," (Cambridge University Press, Cambridge, 1996). 